Case report

\title{
High-resolution computed tomography illustrating pulmonary lymphangitic carcinomatosis in a patient with advanced pancreatic cancer: a case report Liao Wan-Hsiu ${ }^{1}$, Lin Sheng-Hsiang ${ }^{2}{ }^{*}$ and Wu Tsu-Tuan ${ }^{2}$
}

\author{
Address: ${ }^{1}$ Department of Family Medicine, Taipei County Hospital, Taipei County, Taiwan (R.O.C.) and ${ }^{2}$ Department of Internal Medicine, \\ Taipei County Hospital, Taipei County, Taiwan (R.O.C.) \\ Email: LWH - liaowh1976@gmail.com; LSH* - linsh01@gmail.com; WTT - 00291@tpch.gov.tw \\ * Corresponding author
}

Published: 12 May 2009

Received: 6 December 2008

Cases Journal 2009, 2:7428 doi: 10.1/86/1757-1626-2-7428

Accepted: 8 April 2009

This article is available from: http://casesjournal.com/casesjournal/article/view/7428

(c) 2009 Wan-Hsiu et al; licensee Cases Network Ltd.

This is an Open Access article distributed under the terms of the Creative Commons Attribution License (http://creativecommons.org/licenses/by/3.0), which permits unrestricted use, distribution, and reproduction in any medium, provided the original work is properly cited.

\begin{abstract}
We present a case of advanced pancreatic cancer with diffuse pulmonary interstitial infiltrates and dyspnea in a $6 \mathrm{I}$-year-old Asian Taiwanese female. Although surgical lung biopsy is the diagnostic gold standard in most interstitial lung disease, it frequently leads to complications in sick patients. Based on the overall clinico-radiologic correlation, a diagnosis of pulmonary lymphangitic carcinomatosis was supported by the characteristic findings in high-resolution computed tomography.
\end{abstract}

\section{Introduction}

Pulmonary complications are frequently countered in patients with advanced cancer. Although less frequent, the most challenging issue is the so-called "diffuse interstitial infiltrates" on chest radiography. In these patients, the differential diagnosis of various etiologies is difficult. However, the characteristic findings in high-resolution computed tomography (HRCT) are helpful in the diagnosis of lymphangitic carcinomatosis.

\section{Case presentation}

A 61-year-old Asian Taiwanese woman having pancreatic cancer with liver and left adrenal metastases presented with progressive dyspnea for one month. Chest examination revealed crackles at the bases. Cardiovascular examination showed no jugular venous distension and normal heart sounds. An echocardiogram showed normal left ventricular function. A chest radiograph (Figure 1) revealed diffuse reticular opacities and bilateral small amount pleural effusion. HRCT of the lung (Figure 2) showed thickening of peribronchovascular interstitium, interlobular septa, and centrilobular regions. Cytological examination of the pleural fluid revealed adenocarcinoma, most suggestive of a primary origin in the pancreas. However, there was no response to chemotherapy with gemcitabine and she passed away one month later.

\section{Discussion}

The differential diagnosis of dyspnea in a patient with advanced cancer and diffuse interstitial pulmonary infiltrates in chest radiograph is extensive, including viral pneumonia [1], hydrostatic pulmonary interstitial edema [2], chemotherapeutic drugs-induced lung toxicity [3], and lymphangitic carcinomatosis [4]. Since lung biopsies carry a high risk for complications in these patients [5], the characteristic findings of lymphangitic carcinomatosis on 


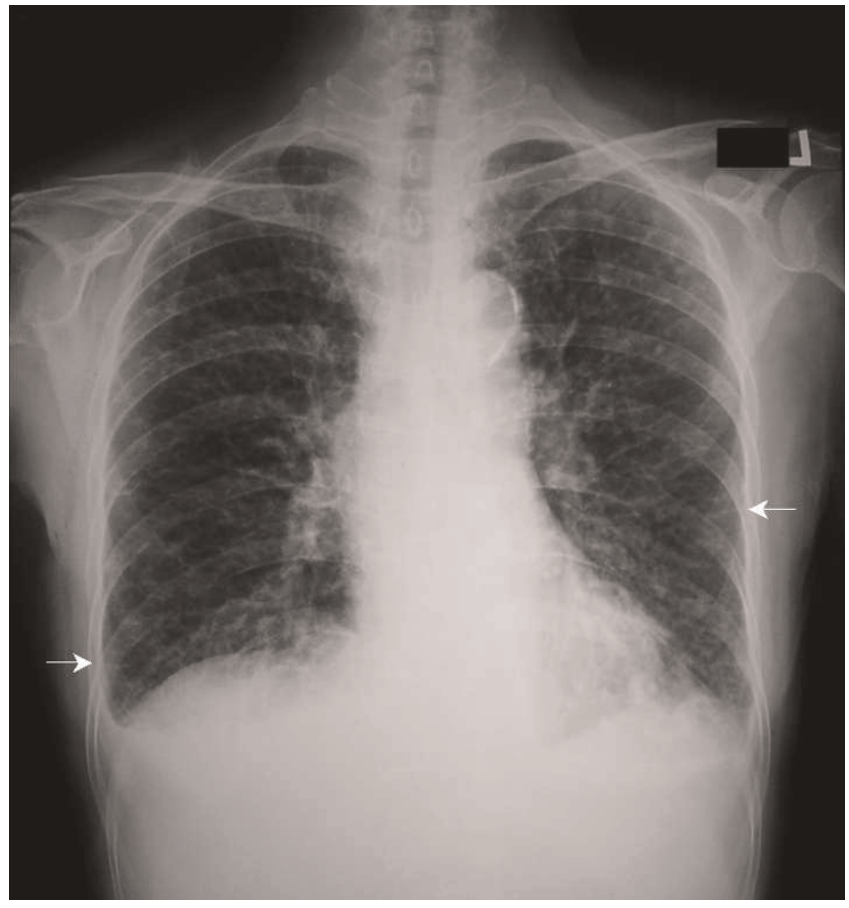

Figure I. Chest radiograph showing diffuse interstitial pulmonary infiltrates with Kerley B lines (arrows) and pleural effusion.

HRCT make great contributions to the differential diagnosis [6].

\section{Conclusion}

In cases with advanced cancer and diffuse interstitial pulmonary infiltrates, HRCT may make contributions to the differential diagnosis, especially while lymphangitic carcinomaotsis is suspected.

\section{List of abbreviations}

HRCT, high-resolution computed tomography.

\section{Consent}

Written informed consent was obtained from the patient for publication of this case report and accompanying images. A copy of the written consent is available for review by the Editor-in-Chief of this journal.

\section{Competing interests}

The authors declare that they have no competing interests.

\section{Authors' contributions}

LWH, LSH and WTT have made significant contributions by making diagnosis and intellectual input in the case and writing the manuscript.

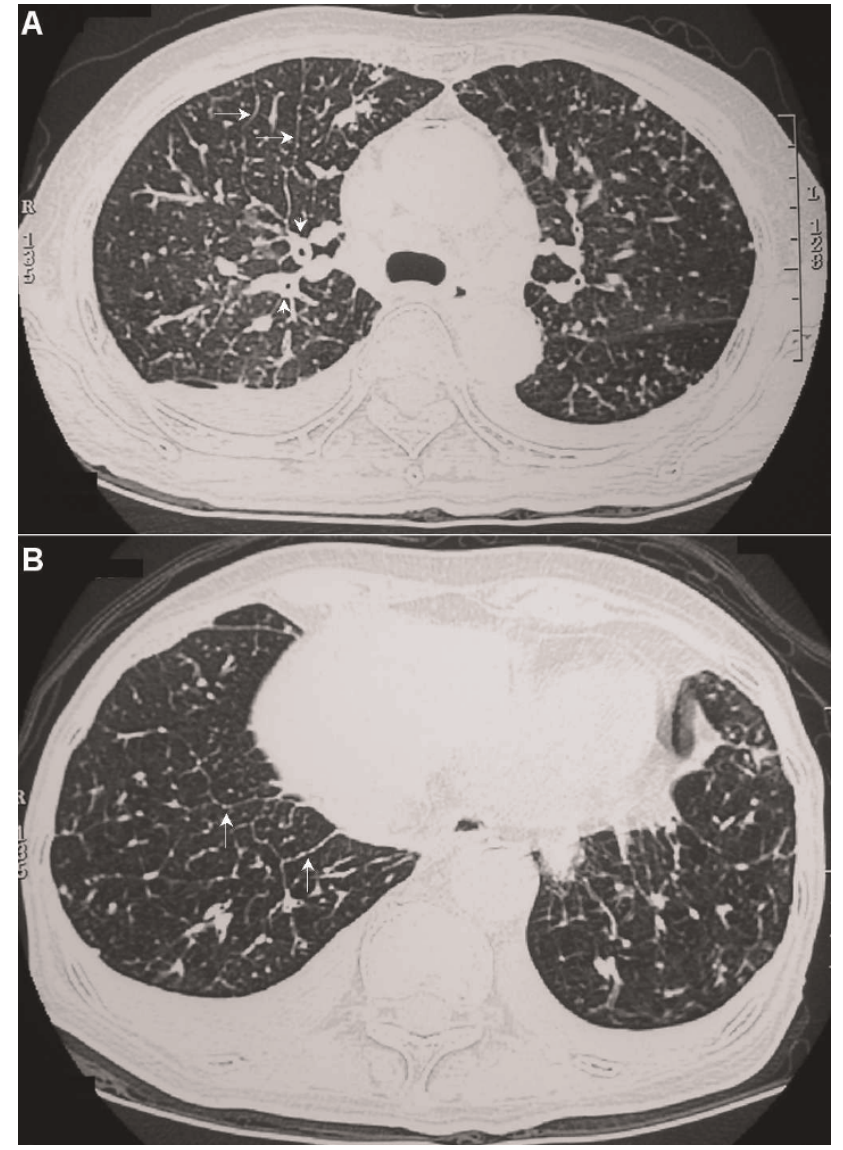

Figure 2. High-resolution chest computed tomography showing (A) Peribronchovascular thickening (arrowheads), irregular thickening of interlobular septa (arrows) and bilateral small amount pleural effusion (B) Interlobular septal thickening forming polygonal arcades (arrow) with prominence of the centrilobular bronchovascular bundle (a central dot in a polygon).

\section{References}

I. Ozbay B, Serto ullarindan B, Tekin M, Altinoz O: Influenzaassociated pneumonia in a Turkish area with endemic avian influenza. Respirology 2008, I 3:444-446.

2. Ribeiro CM, Marchiori E, Rodrigues R, Gasparetto E, Souza AS Jr, Escuissato D, Nobre LF, Zanetti G, de Araujo Neto C, Irion K: Hydrostatic pulmonary edema: high-resolution computed tomography aspects. J Bras Pneumol 2006, 32:5I5-522.

3. Shaib W, Lansigan F, Cornfeld D, Syrigos K, Saif MW: Gemcitabineinduced pulmonary toxicity during adjuvant therapy in a patient with pancreatic cancer. JOP 2008, 9:708-7/4.

4. Thomas A, Lenox R: Pulmonary lymphangitic carcinomatosis as a primary manifestation of colon cancer in a young adult. CMAJ 2008, I 79:338-340.

5. Solaini L, Prusciano F, Bagioni P, di Francesco F, Solaini L, Poddie DB: Video-assisted thoracic surgery (VATS) of the lung: analysis of intraoperative and postoperative complications over I5 years and review of the literature. Surg Endosc 2008, 22:298-3 I0.

6. Castañer E, Gallardo X, Pallardó Y, Branera J, Cabezuelo MA, Mata JM: Diseases affecting the peribronchovascular interstitium: $C$ T findings and pathologic correlation. Curr Probl Diagn Radiol 2005, 34:63-75. 